Article

\title{
Cloning and Functional Assessments of Floral-Expressed SWEET Transporter Genes from Jasminum sambac
}

\author{
Panpan Wang ${ }^{1}$, Peining Wei ${ }^{2}$, Fangfei Niu ${ }^{1}$, Xiaofeng Liu ${ }^{1}$, Hongliang Zhang ${ }^{1}$, Meiling Lyu ${ }^{1}$, \\ Yuan Yuan ${ }^{1}$ and Binghua $W^{1}{ }^{1, *}$ D \\ 1 Fujian Provincial Key Laboratory of Plant Functional Biology, College of Horticulture, Fujian A \& University, \\ Fuzhou 350002, China \\ 2 College of Life Sciences, Fujian Agriculture and Forestry University, Fuzhou 350002, China \\ * Correspondence: binghua.wu@fafu.edu.cn
}

Received: 19 July 2019; Accepted: 14 August 2019; Published: 16 August 2019

\begin{abstract}
Sugar transporters of the SWEET family mediate cross membrane movement of monoand disaccharides and play vital roles in diverse physiological and pathophysiological processes, including sink-source relationship, pathogen responses, reproductive growth, and development. However, it remains to be determined how these transporters function in non-module plants of agricultural significance, given the evolutionarily diverse traits. In this study, we combined transcriptome analysis, rapid amplification of cDNA ends-cloning (RACE-cloning), expression profiling, and heterologous functional assay to identify SWEET genes that may have potential roles during flower opening and sexual reproduction in Jasminum sambac. During the anthesis, the floral organs of J. sambac express seven SWEET homologous genes from all four clades of the family. JsSWEET9 and 2 are significantly upregulated when flowers are fully opened, up to 6- and 3-fold compared to unopened buds, respectively. The other transporters, JsSWEET1, 5, 10, and 17 are also accumulated slightly at stage associated with fragrance release, whereas only the vacuole transporter JSSWEET16 showed small decrease in transcript level after anthesis. The JsSWEET5, a clade II member, is capable to complement yeast cell uptake on most tested sugar substrates with a preference for hexoses, while the clade I transporter JSSWEET1 mediates merely galactose import when expressed in yeast. Our results provide first evidence for further investigation on sugar transport and allocation during flowering and reproductive processes in J. sambac.
\end{abstract}

Keywords: SWEET transporter; gene expression; sugar transport; flowers; Jasminum sambac

\section{Introduction}

Sugar transport in higher plants involves at least three major groups of proteins [1]. Members of the monosaccharide/polyols transporters-like (MST) superfamily constitute seven divers subfamilies and transport a wide range of substrates in addition to glucose [2-4]. For example, the subfamily of Sugar Transport Proteins (STP) in Arabidopsis contains fifteen genes, six out of which have been shown to act redundantly in mediating glucose uptake by pollen tubes $[5,6]$. More importantly, members of the STPs-AtSTP1 and AtSTP13 - have been implicated in antibacterial defense by sequestration of apoplasmic sugars to limit pathogens' nutrition $[7,8]$. Another major class of transporters belongs to the sucrose transporter/carriers (SUT/SUC) family, which are the first sucrose/ $\mathrm{H}^{+}$symporters characterized as being important for phloem loading and unloading processes [9]. This family has nine genes in Arabidopsis, but typically consists of five members in haploid of most monocotyledon species as well as in ancient vascular plants such as Selaginella, and one single gene in moss [2]. 
Homologs of the SUT/SUC family can be classified phylogenetically as three types or five clades [10,11], with the existence of evolutionary isolated subclade members. Many SUTs have demonstrated sucrose and/or maltose transport activities at plasma membrane or tonoplast [11]. Being major players for photosynthetic sucrose translocation and partitioning between source-sink organs, most of the SUT transporters are expressed mainly in phloem tissues of both source (leaves) and sink (roots, flowers, and other storage or reproductive structures) organs, especially in the sieve element-companion cell complex [10,12]. Both MST and SUT/SUC families are closely related to the major facilitator superfamily (MFS).

The third group of sugar transporters identified are the SWEET proteins that mediated bidirectional cross-membrane movement of mono- and disaccharides by an alternating access mechanism [13-16]. The eukaryotic SWEET proteins have a conserved trimeric structure with seven transmembrane helices that fold into two symmetric $\mathrm{N}$ - and C-terminal bundles (triple-helix bundles, THBs), linked by the fourth helix, whereas their prokaryotic counterparts, called SemiSWEETs that also function as monoor disaccharides transporters, contain only a single THB enduring a dimeric structure [17-20]. In general, a typical angiosperm genome contains 15 to 25 SWEET genes, e.g., 17 are encoded in Arabidopsis and 21 in rice. SWEET gene duplication occurs during evolution in numerous plant species, such as Eucalyptus (47 genes) [14], soybean (52) [21], wheat (108) [22], cabbage (30) [23], cultivated cotton (55) [24], and rubber tree (36) [25], contrary to only one single gene encoded by human and Drosophila genomes $[16,26]$. The four-clade classified SWEET proteins play very important roles in diverse physiological and pathophysiological processes (recent reviews in $[14,27,28])$. The involvements of different SWEET homologs in source-sink relationship [15,29], pathogen responses [16,30,31], nectar secretion [32], and fruit/seed development [33-35] are among the well-studied.

The reproductive organs of higher plants, from anthesis to seed maturation, are temporally and spatially strong sinks for sugar allocation and distribution. The importance of sugar transport for the reproduction process is highlighted by the expression of as many as 31 distinct sugar transporter genes in Arabidopsis floral organs [36], and individual transporter may also have critical function. For example, lack of AtSWEET8 (also called RPG1) leads to male sterility [37]. AtSWEET9 functions essentially as a sucrose efflux transporter in the nectaries, together with sucrose synthase (SUS) and cell wall invertases (cwINV), for nectar secretion of a mixture of sugars. Interestingly, silencing the Petunia homolog of SWEET9, PhNEC1, resulted in malfunction of anther opening and male sterility [38]. However, most of our knowledge about the transport, distribution, metabolism, and signaling of sugars in the reproduction processes are gained from studies in model plants such as Arabidopsis, whilst other plant species may have evolved other mechanisms.

Jasminum sambac is a fragrant ornamental plants widely cultivated in the Middle East and South-East Asia for harvest of flowers. Under nature conditions the plant exhibit a very low fruit and seed set ranging from $0.13 \%$ to $0.19 \%$ due to low pollen viability and possible premature cessation of pistil development that hinders the breeding efforts for variety improvement [39,40]. Despite the horticultural significance, it has not been fully exploited at the molecular level regarding the sexual reproduction of this plant species. Carbohydrate metabolism and regulation during flower growth are among the important issues to be addressed [36]. In this study, we identified seven floral expressed SWEET transporter genes through transcriptome analysis and RACE-cloning. By monitoring their expression during anthesis and characterizing their uptake activity in a yeast complementation assay, we showed that JSSWEET2 and JSSWEET9 were the most expressed genes at the later stage of flowering and the JSSWEET5 was able to transport both mono- and disaccharides at the plasma membrane. Our results provides useful information for further deciphering functions of sugar transporters during flowering in this special plant. 


\section{Results}

\subsection{Identification of Seven Flower-Expressed SWEET Genes Representative of the Four-Clade Family}

As a fragrant and nocturnal flowering plant, J. sambac has no genomic sequences available yet, despite its horticultural significance. We first conducted a transcriptome analysis with flower and leaf samples at three time points during flower opening (Genebank accession GHOY00000000). In the flower transcriptome, seven SWEET transporter-like, four SUC/SUT transporter-like and a number of the monosaccharide transporters-like (belong to MST family) unigenes, together with homologous genes encoding invertases and trehalose-6-phosphate synthases, are differently expressed at the time-points during the day (Figure 1). Flowers of J. sambac normally open right after the dusk and release fragrance at mid night, and last for approximately 26 to $30 \mathrm{~h}[40,41]$. Our transcriptome roughly covers gene expressions during the major stages of flowering. These data provide an overview on important sugar utilization-related gene expression in the sink tissues (Figure 1). Specifically for the SWEET homologous genes, five of the seven ESTs were not found in leaves at 17:00 and 05:00, suggesting a floral-specific function (Table 1).

Table 1. Unigenes of SWEET homologs preferentially expressed in flowers identified from a transcriptome analysis.

\begin{tabular}{ccccccc}
\hline Unigene_id & $\begin{array}{c}\text { Flowers } \\
\text { @11:00 } \\
\text { (FPKM) }\end{array}$ & $\begin{array}{c}\mathbf{1} \\
\mathbf{\text { Flowers }} \\
\text { @17:00 } \\
\text { (FPKM) }\end{array}$ & $\begin{array}{c}\text { Flowers } \\
\text { @5:00 } \\
\text { (FPKM) }\end{array}$ & $\begin{array}{c}\text { Leaves } \\
\text { @17:00 } \\
\text { (FPKM) }\end{array}$ & $\begin{array}{c}\text { Leaves } \\
\text { @5:00 } \\
\text { (FPKM) }\end{array}$ & $\begin{array}{c}\text { Swissprot } \\
\text { Evalue }\end{array}$ \\
\hline c41111_g1 & 2.775 & 2.435 & 2.035 & 0 & 0 & $1.6 \times 10^{-4}$ \\
c50045_g1 & 15.92 & 14.29 & 11.59 & 0 & 0 & $1.7 \times 10^{-71}$ \\
c51046_g2 & 2.655 & 6.975 & 39.445 & 0 & 0 & $6.4 \times 10^{-86}$ \\
c51507_g1 & 14.165 & 20.89 & 43.425 & 0 & 0 & $9.6 \times 10^{-106}$ \\
c44769_g1 & 36.935 & 72.54 & 28.14 & 0 & 0.075 & $2.07 \times 10^{-66}$ \\
c42726_g1 & 4.95 & 11.43 & 9.86 & 13.32 & 2.62 & $1.2 \times 10^{-56}$ \\
c60775_g2 & 55.56 & 36.72 & 106.135 & 7.59 & 7.755 & $4.8 \times 10^{-87}$ \\
\hline
\end{tabular}

${ }^{1}$ FPKM: expected number of Fragments Per Kilobase of transcript sequence per Millions base pairs sequenced.

Using sequences of the seven SWEET ESTs (Table 1) as guidance, we designed primers and amplified their full-length cDNA via $5^{\prime} / 3^{\prime}$-RACE PCR. With this strategy, we successfully obtained a set of SWEET genes that were preferentially expressed, and might play substantial functions, during the blossom of J. sambac.

Sequence analysis revealed that these proteins represent all the four clades of the SWEET family (Figure 2 and Figure S1). Accordingly, we renamed the full-length cDNA to JsSWEET1 (c50045_g1), JsSWEER2 (c60775_g2), JsSWEET5 (c41111_g1), JsSWEET9 (c51507_g1), JsSWEET10 (c44769_g1), JsSWEET16 (c51046_g2), and JsSWEET17 (c42726_g1), respectively. JsSWEET1 shares $71.37 \%$ amino acid identity with its arabidopsis orthodox AtSWEET1, followed by JsSWEET5 with $62.87 \%$ to AtSWEET5. The JsSWEET2 is more closely related to AtSWEET2 (60.43\%) than to OsSWEET2b $(53.95 \%)$ (Table S4). 


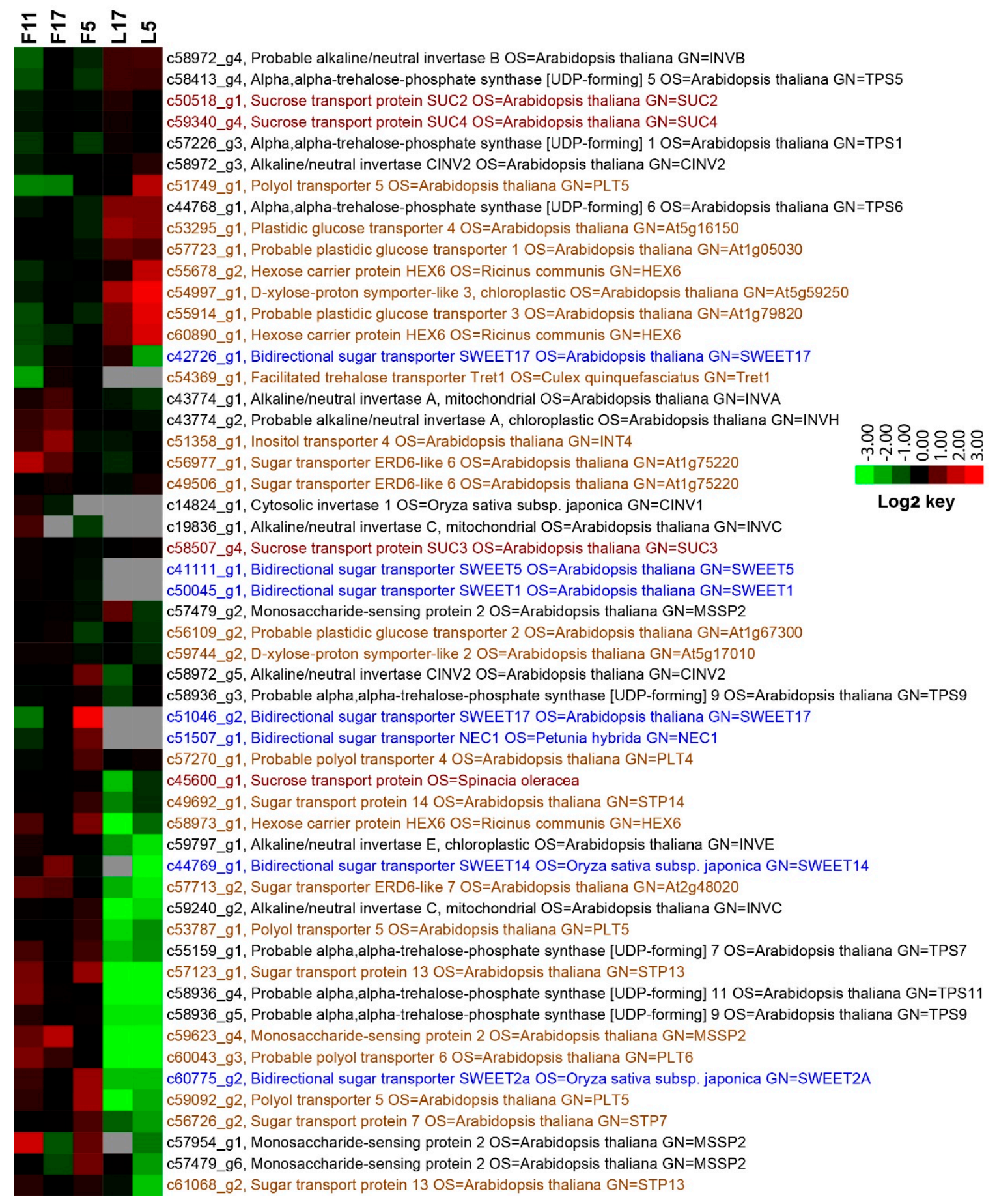

Figure 1. A heat map showing differentially expressed genes for SWEET transporter-like (highlight in blue), sucrose transporter-like (in red) and monosaccharide transporter-like (in orange), invertases-like and trehalose-6-phosphate synthase-like in J. sambac flowers and leaves at indicated time of the day. Genes were clustered by uncentered Pearson correlation with average linkage ordering, using Cluster 3.0 (version 1.57). The $\log _{2}$-transformed fold change is indicated by the color bar. Samples F11, F17, F5, and L17, L5: flowers at 11:00, 17:00, 05:00, and leaves at 17:00, 05:00. 


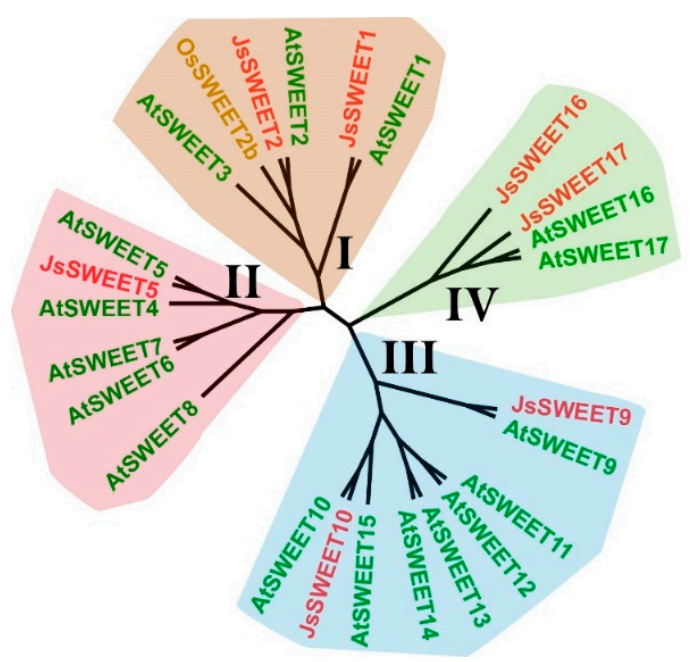

Figure 2. A phylogenetic tree showing the sequence relationship between the seven cloned J. sambac SWEET transporters (in red) and the seventeen members from Arabidopsis (in green), together with the rice homolog, OsSWEET2b (in yellow), which has a solved structure (PDB ID: 5CTH) available.

\subsection{Expression of the SWEET Genes during Flower Opening Stages}

The flower buds of J. sambac open at approximately 19:00 in the evening and remain opening during the next 26 to $30 \mathrm{~h}$. The first $12 \mathrm{~h}$ is considered the most important period for fragrance emission (our unpublished data, [41]) and pollination [40]. To monitor the SWEET gene expression during the processes, we isolated RNA form flowers at four time points of the day at 14:00 (unopen flower buds), 17:00 (initiating loose buds), 23:00 (opening), and 5:00 (fully open) (Figure 3) and measured the relative mRNA levels using quantitative RT-PCR.

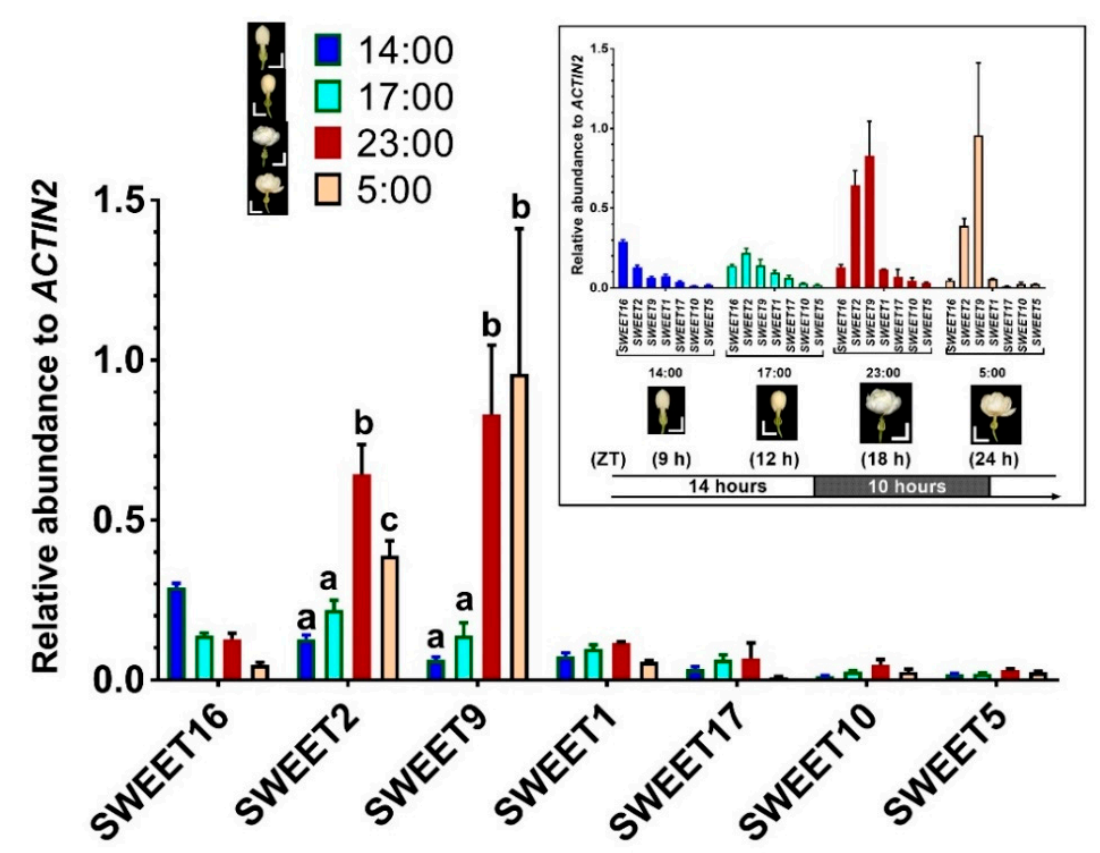

Figure 3. SWEET gene expressions during J. sambac flower opening. Transcript abundance relative to JsACTIN2 was determined using quantitative RT-PCR in flower samples collected at indicated time points representing four floral stages. Significant difference between samples is indicated by different letters above the bars, which was conducted using the two-way ANOVA and multiple T-test implemented in the Prism software (version 7.04). The insert rearranges the data by the sampling 
time points. To correlate the time points with the circadian light/dark rhythm, the corresponding Zeitgeber time $(\mathrm{ZT})$ was given below the sampling stage. Data are mean $\pm \mathrm{SD}(n=3$ biological replicates). Scale bars $=1 \mathrm{~cm}$.

JSSWEET10 and JsSWEET5 were the lowest expressed genes in the SWEET family, while JsSWEET2 and JsSWEET9 had the most abundant transcript levels (Figure 3). This significantly up-regulated expression of JsSWEET9 during anthesis was similar to its orthologues in Arabidopsis [32] and Petunia [42]. Furthermore, the expression of JSSWEET16 seemed to decrease after flower opening, reaching one-sixth at time of fully-open to that in bud stage. It was the only gene that showed a down-regulated pattern (Figure 3). Otherwise, the highly expressed transcripts of JSSWEET9 and JSSWEET2 were observed in fully opened flowers (Figure 3). The other four genes-namely JsSWEET1, JSSWEET17, JSSWEET10, and JSSWEET5-were expressed at relatively low level, all of which were maintained in a more or less stable fashion during the flower opening (Figure 3).

\subsection{Subcellular Localization of the Seven SWEET Proteins}

Functions of a membrane transporter depend very much on its subcellular localization. Using transient expressions of a C-terminal in-frame fusion with the green fluorescence protein (GFP), we determined the subcellular localization of the SWEET proteins in protoplasts isolated from petals of J. sambac flowers, as well as in arabidopsis leaf protoplasts and Nicotiana benthamiana epidermis cells.

JsSWEET5 clearly showed a plasma membrane (PM) localization in both types of protoplasts and also in tobacco epidermis, whereas both JsSWEET9 and JsSWEET10 were localized to PM but with spot-like particles in cytosolic regions close to the PM. These spot-like particles were supposed to be Golgi or ER-PM-contact structures (Figures 4 and 5). In both protoplast expressions, JsSWEET2 could be localized to the tonoplasts, but that was difficult to confirm in tobacco epidermis (Figures 4 and 5). The JsSWEET1 apparently showed a PM localization but also displayed some strong intracellular signals in petal protoplasts (Figure 4), it was not expressed well in the arabidopsis leaf protoplasts. Both JsSWEET16 and 17 exhibited cytosolic or vacuolar distributions in protoplasts and in N. benthamiana epidermis (Figures 4 and 5). JsSWEET17 was also not expressed well in arabidopsis leaf protoplasts. 


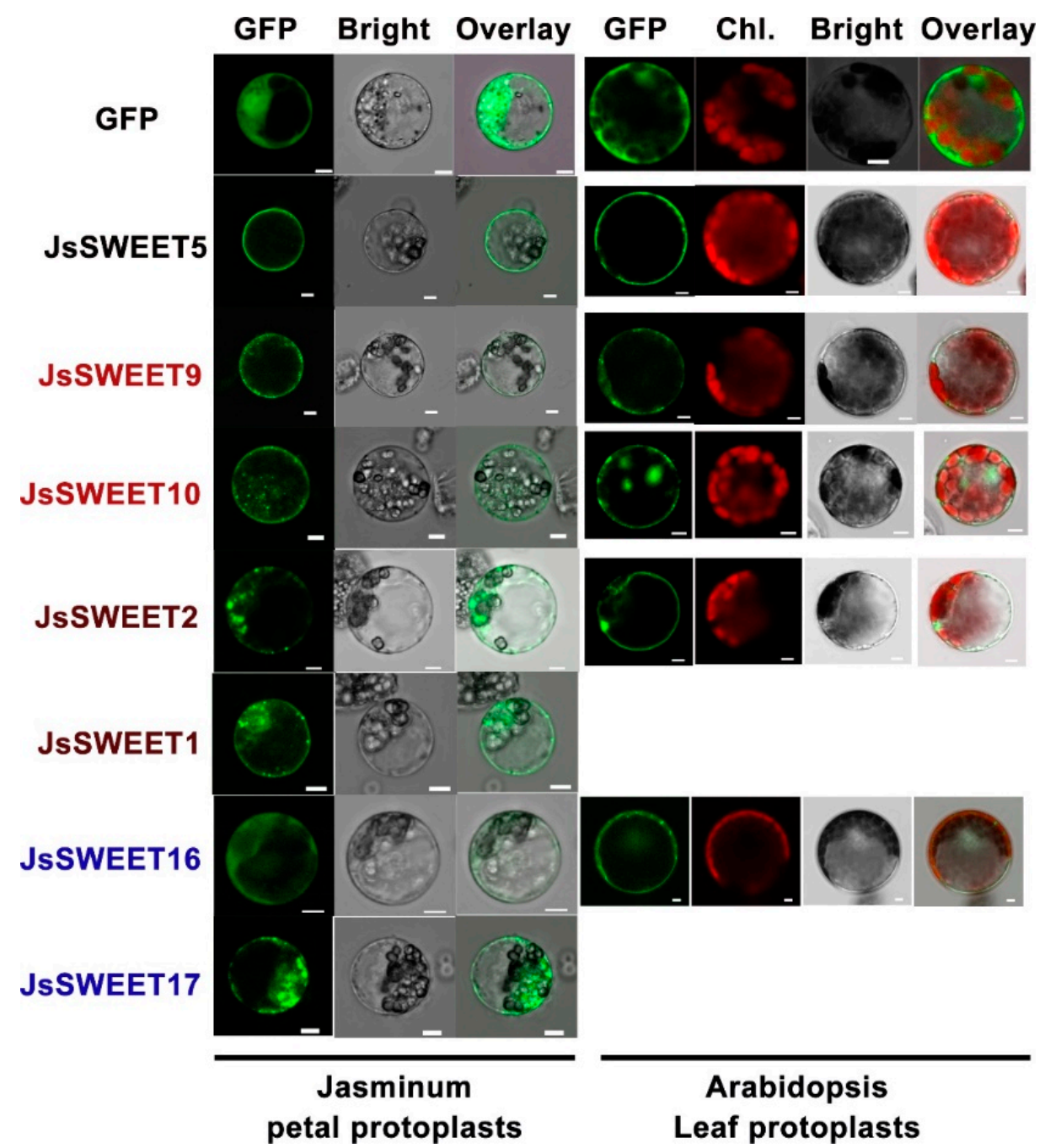

Figure 4. Confocal images of SWEET-GFP (green fluorescence protein) fusion proteins transiently expressed under $35 \mathrm{~S}$ promoter in protoplasts of $J$. sambac petals and arabidopsis leaves. Images were acquired using a Leica TCS SP8 system with wavelength setting for GFP $(E x=488 \mathrm{~nm}$, Em $=507 \mathrm{~nm})$ or chlorophyll $(\mathrm{Ex}=488 \mathrm{~nm}, \mathrm{Em}=681 \mathrm{~nm})$. Gene names are colored for clade-specific members (brown for Clade I, black for Clade II, red for Clade II and blue for Clade IV). JsSWEET1 and JsSWEET17 failed to express well in arabidopsis leaf protoplasts. Scale bar $=5 \mu \mathrm{m}$.

Except for JsSWEET16 and JsSWEET17, the transient expression of the GFP-fusions for the other five genes in N. benthamiana epidermis displayed apparent PM localization signals (Figure 5). These observation suggested that the subcellular localization of the SWEET proteins were sometime cell-type dependent, likely reflecting a dynamic trafficking feature for members of the family. 


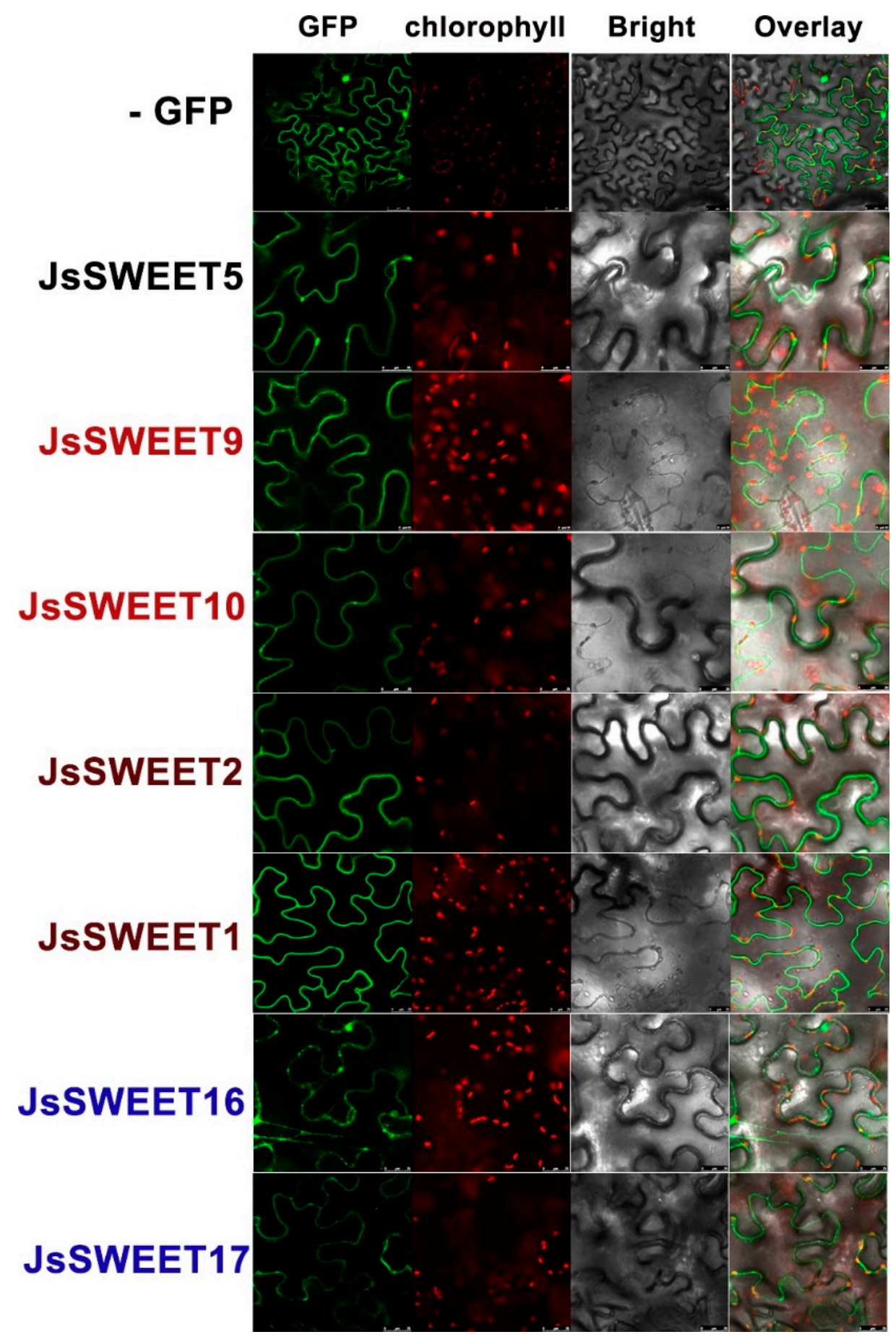

Figure 5. Transient expression of JsSWEET-GFP fusion proteins driven by the $35 \mathrm{~S}$ promoter in $N$. benthamiana epidermis. Images were acquired using a Leica TCS SP8 system with wavelength setting for GFP $(E x=488 \mathrm{~nm}, \mathrm{Em}=507 \mathrm{~nm})$ or chlorophyll $(E x=488 \mathrm{~nm}, \mathrm{Em}=681 \mathrm{~nm})$. Gene names colored for clade-specific members (brown for Clade I, black for Clade II, red for Clade II, and blue for Clade IV). Scale bars, 25 (GFP control) or 50 (the rest) $\mu \mathrm{m}$.

\subsection{The JsSWEET5 Is a Multi-Substrates Transporter Revealed by a Yeast Complementation Assay}

The yeast strain EBY4000 is defected for its endogenous 20 sugar transporter genes (17 from hexose transporter family, 1 for galactose transporter and 2 for maltose permeases), thus incapable to support cell growth on most sugar media except for maltose [43]. We expressed the cloned JSSWEET genes individually in this strain and examined their complementation for uptake of different sugars. 
The yeast transformants of JsSWEET16, 17, and 2 were not able to complement the growth defect on all the sugar media tested, probably due to their vacuole or intracellular localizations (Figure 6). Similarly, expression of both JsSWEET10 and JsSWEET9 did not permit the uptake of sugars tested, indicating that either they were not properly targeted to the PM or that they might function solely as efflux transporters, as demonstrated for the arabidopsis homologue AtSWEET9 [32]. Further, in the yeast medium containing 2-deoxyglucose (2-DOG, a yeast toxic analog of glucose), yeast cells with a functional hexose importer would display a growth inhibition. Yeast cells harboring expression vectors for the above five JSSWEETS were not sensitive to 2-DOG (Figure 6). However, the JSSWEET1-expressing yeast cells showed only limited sensitivity to 2-DOG. This transformed yeast was able to grow weakly on galactose medium, meaning that JsSWEET1 was a weak galactose transporter (Figure 6).

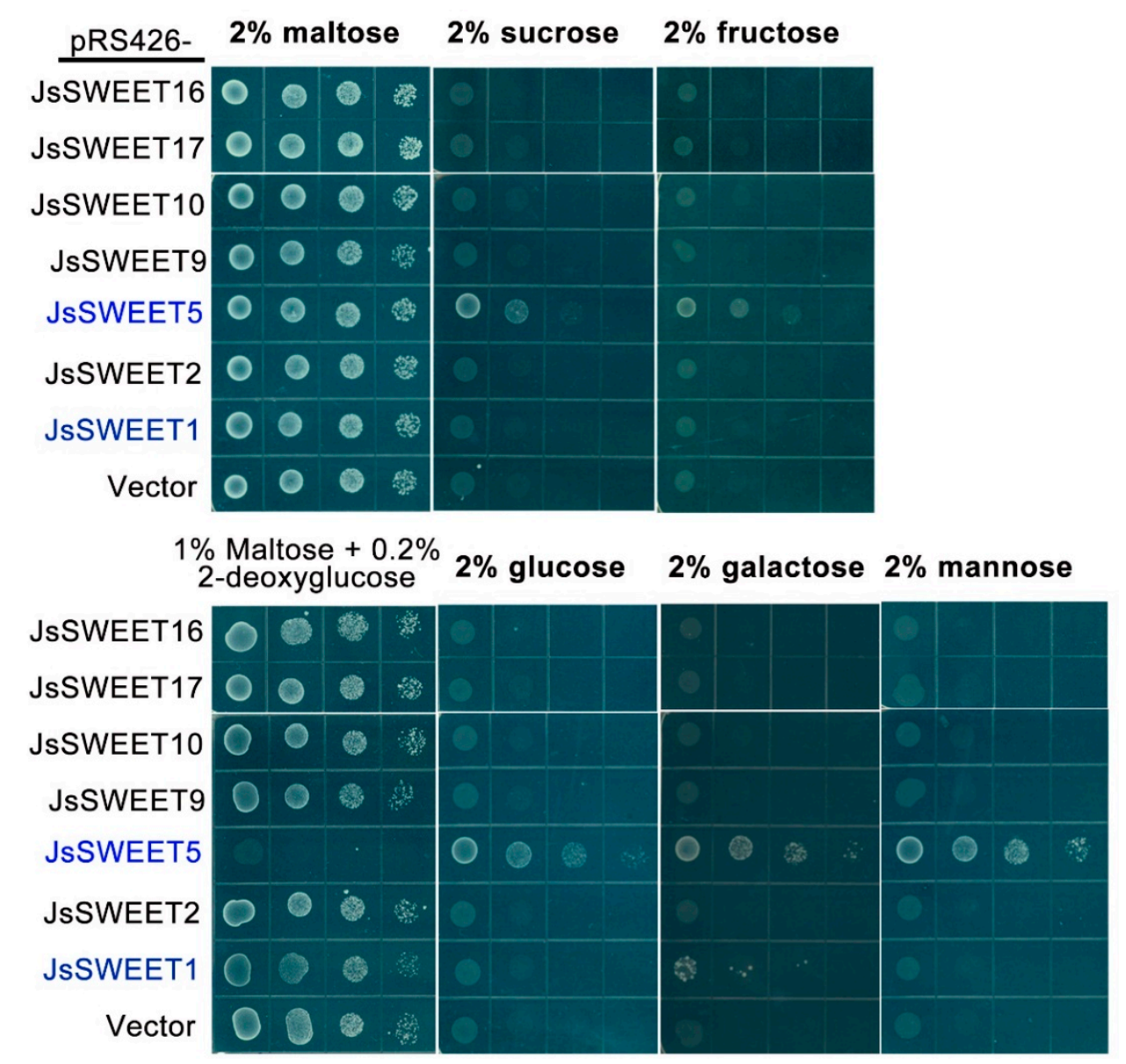

Figure 6. Growth complementation assay in yeast deficient strain EBY4000 by the expression of the JSSWEETs. Ten microliter each of a serial 10-fold diluted cell suspensions were dropped in selection media containing $0.67 \%$ Difco yeast nitrogen base with ammonium sulfate supplemented with leucine, tryptophan, histidine, and the indicated carbon sources). Maltose medium serves as a growth control for plasmid maintaining. Note that only yeast cells expressing JsSWEET1 and JsSWEET5 (highlighted in blue) showed growth complementation.

JsSWEET5 was cable to complement the growth defect on all five sugar media-with the most on mannose and less on sucrose-and its expression rendered the yeast cells much sensitive to 2-DOG (Figure 6). Therefore, JsSWEET5 was a PM sugar transporter with a preference for hexoses over disaccharides. 


\section{Discussion}

Sucrose is the predominant sugar for long-distance translocation. Being imported from the terminal phloem around the base of the flowers, sucrose is first unloaded by at least the Clade III SWEETs into the apoplasmic space, where it may be distribute to different nearby floral tissues or partially converted by cwINV to glucose and fructose. Re-uptake and transport of these sugars in the symplast among petals, androecium and gynoecium are specifically controlled by various sugar transporters. Transport activities are spacialtemporally controlled to correlate with flower development and metabolic needs. Petals are sites to attract pollinators by synthesis of colored and/or volatile secondary metabolites, by production of nectary secretion with sugar and amino acids, and importantly by generation of an osmotic-driven flower opening. All of which are sugar demanding processes [36]. To ensure successful male fertility, it has been known that specific sugar transporters are required during anthers, pollen grain development, pollen germination and pollen tube growth [44]. Furthermore, fertilization and embryo development involve intensive metabolic and signaling activities that are relevant to the functions and regulations of sugar transporters [33,36,45].

As a nocturnal pollination plant, J. sambac has a relatively short time-scale for floral scent production in the middle of the night [41] (our unpublished data). During this time two SWEET genes, JsSWEET9 (Clade III) and JsSWEET2 (Clade I) are up-regulated, their expression remain at very high levels throughout till dawn (Figure 3). Although not yet proved, JsSWEET9 may be a candidate involved in nectary secretion during the flower opening, as similar to the so-far-demonstrated role of the arabidopsis orthologue AtSWEET9 [32,42]. Orthologues of JsSWEET2 in arabidopsis and rice are known to function as tonoplast glucose transporters important for glucose sequestration in the vacuole, which is the major compartment for sugar storage [17,31]. JsSWEET2 shares 54-60\% identity at the amino acids level with these two proteins and is also tonoplast localized when transiently expressed in protoplasts (Figure 4). Its expression in yeast fails to complement the uptake of glucose and other hexose or sucrose, and renders insensitive to the toxic glucose analogue 2-DOG as well (Figure 6). Thus, we speculate that JsSWEET2 may function in vacuole sugar mobilization. It will be necessary, however, to further determine its tissue or cell-type specific expression and knockout phenotype. Another Clade III member JsSWEET10 is a putative transporter in sucrose unloading.

The Clade IV members JsSWEET16 and 17 are tonoplast transporters (Figures 4 and 5), their homologous in Arabidopsis play pivotal roles in controlling vacuole fructose storage [46-48]. JSSWEET16 seems to be expressed more in floral bud stage whereas JSSWEET17 maintains low but stable expression during anthesis (Figure 3). Whether they are involved in transient sugar storage or remobilization will await further studies.

The flowers of J. sambac also express another Clade I member, JsSWEET1 and a single Clade II gene JsSWEET5, and both encode plasma membrane localized proteins. JsSWEET1 shows weak transport for galactose but rejects glucose or fructose. For a comparison, the AtSWEET1 could mediate weak transport of mannose, fructose, and galactose, in addition to its pH-dependent uptake of glucose [16]. JsSWEET5 is able to complement the yeast uptake of various sugars with stronger preference for hexose (Figure 6). The rice orthologue OsSWEET5 is expressed in anthers [49] and the AtSWEET5 (also called AtVEX, VEGETATIVE CELL EXPRESSED1) promoter-reporter translational fusions are found in mature, hydrated and germinating pollen [50]. Therefore, JsSWEET5 is very likely a sugar transporter of reproduction-relevance in J. sambac. Both JsSWEET1 and 5 have similar expression profiles during flowering (Figure 3), it will be interesting to further delineate their cell- or tissue-specific roles in J.sambac.

In summary, using floral transcriptome data as a guide, we have identified seven SWEET transporters expressed in flowers of J. sambac, which are well represented by the four clades of the SWEET family. Further investigations on their specific functions during reproductive development will provide helpful knowledges for crop improvement of this particular species, and is of general interests in biology of sugar transport as well. 


\section{Materials and Methods}

\subsection{Plant Materials and Growth Conditions}

Three-year-old J. sambac var "double petals" plants were cultivated in pots $(25 \times 25 \times 30 \mathrm{~cm})$ and maintained in climate room, with $14 \mathrm{~h}$ photoperiod (light on at 05:00 and off at 19:00) under LED illumination (12-15 kLux) and temperature of $28 / 22^{\circ} \mathrm{C}$. Under our conditions, flowering usually started at c.a. 19:00 of the day, and last for the next 30 to $36 \mathrm{~h}$. Flower buds were individual labeled two days priors to sampling to insure a unified developmental stage.

\subsection{RNA-seq Library Preparation and Transcriptome Generation}

For RNA isolation, leaves at 17:00 and 05:00 and flowers at 17:00, 05:00, and 11:00 were collected from three to five plants, immediately immersed into liquid nitrogen and kept in $-80^{\circ} \mathrm{C}$. Samples of five leaves, or five to eight flowers, were pooled as one biological replication. Two biological replications were used for transcriptome library preparations, representing a total of 20 plants. Approximately $1 \mathrm{~g}$ of fresh tissue was ground into fine powders in liquid nitrogen, and the frozen powders were used for total RNA extraction with a commercial kid (Trizol extraction kit from Invitrogen). RNA degradation and contamination was monitored on $1 \%$ agarose gels and the purity was checked using the Nano Photometer ${ }^{\circledR}$ spectrophotometer (IMPLEN, Westlake Village, CA, USA). The RNA integrity was assessed using the RNA Nano 6000 Assay Kit of the Agilent Bioanalyzer 2100 system (Agilent Technologies, Santa Clara, CA, USA).

A total amount of $1.5 \mu \mathrm{g}$ RNA per sample was used as input material for the RNA sample preparations. Sequencing libraries were generated using NEBNext ${ }^{\circledR}$ Ultra ${ }^{\mathrm{TM}}$ RNA Library Prep Kit for Illumina ${ }^{\circledR}$ (NEB, Ipswich, MA, USA) following manufacturer's recommendations and index codes were added to attribute sequences to each sample. Briefly, mRNA was purified from total RNA using poly-T oligo-attached magnetic beads. Fragmentation was carried out using divalent cations under elevated temperature in NEB Next First Strand Synthesis Reaction Buffer (5x). First strand cDNA was synthesized using random hexamer primer and M-MuLV Reverse Transcriptase (RNase $\mathrm{H}^{-}$). Subsequently second strand cDNA synthesis was performed using DNA Polymerase I and RNase $H$. Remaining overhangs were converted into blunt ends via exonuclease/polymerase activities. After adenylation of $3^{\prime}$ ends of the DNA fragments, adaptor with hairpin loop structure (NEB Next Adaptor, from NEB, Ipswich, MA, USA) were added by ligation. These cDNA fragments were size-selected for 150 200 bp in length, and purified with AMPure XP system (Beckman Coulter, Beverly, MA, USA). Prior to PCR amplification, $3 \mu \mathrm{L}$ USER Enzyme (NEB, Ipswich, MA, USA) was used to treat the size-selected, adaptor-ligated cDNA at $37^{\circ} \mathrm{C}$ for $15 \mathrm{~min}$ followed by $5 \mathrm{~min}$ at $95^{\circ} \mathrm{C}$ PCR. The PCR was performed with Phusion High-Fidelity DNA polymerase, Universal PCR primers and Index $(X)$ Primer. Amplified PCR products were purified with an AMPure XP system. Finally, the quality of library was assessed on the Agilent Bioanalyzer 2100 system.

After cluster generation (using cBot Cluster Generation System of the TruSeq PE Cluster Kit v3-cBot-HS from Illumia), the library preparations were sequenced on an Illumina Hiseq platform and paired-end reads were generated. The cleaned row reads have been deposited at DDBJ/ENA/GenBank under the Accession SRR9613490 through SRR9613499. The transcriptome was assembled using Trinity [51]. Differential expression analysis was performed using the DESeq $R$ package (1.10.1). Q value $<0.005$ and $\log 2$ (fold-change) $>1$ was set as the threshold for significantly differential expression [52]. Gene Ontology (GO) enrichment analysis of the differentially expressed genes (DEGs) was implemented by the GOseq R packages based Walleniusnon-central hyper-geometric distribution [53] which can adjust for gene length bias in DEGs. The generated Transcriptome Shotgun Assembly project has been deposited at DDBJ/ENA/GenBank under the accession GHOY00000000. The version described in this paper is the first version, GHOY01000000. 


\subsection{Full-Length cDNA Cloning and Sequence Analysis}

Full-length cDNAs of the seven SWEET genes were PCR cloned using the SMARTer RACE $5^{\prime} / 3^{\prime}$ Kit from TaKaRa Bio with gene-specific primers (listed in Supplemental Table S1) designed based on the transcriptome sequencing data. Complete sequences with UTR were cloned into the Gateway vector pENTYR ${ }^{\circledR}$-/D-TOPO (Thermo Fisher Scientific, Shanghai, China) and subjected to Sanger sequencing. For phylogenetic analysis, sequences of the Arabidopsis 17 SWEET and the rice OsSWEET2b were download from NCBI database. Amino acid sequence alignment was conducted using MUSCLE (https://www.ebi.ac.uk/Tools/msa/muscle/) and the Neighbor-Joining tree was generated using MEGA X (https://www.megasoftware.net/) with a bootstrap number 1000.

\subsection{Reverse Transcription Quantitative PCR (RT-PCR)}

Total RNA was isolated from flower buds (at 14:00 and 17:00 of the first day) and opened flowers (at 23:00 of the first day and 05:00 of the next day) using an RNAprep pure Kit (TIANGEN Biotech, Beijing China). First-strand cDNA synthesis was performed with the TransScript One-Step gDNA Removal and cDNA Synthesis SuperMix (TransGen, Shanghai, China). Quantitative PCR was run on a Roche LightCycler 96, using a PCR program set as an initial step of $30 \mathrm{~s}$ at $94{ }^{\circ} \mathrm{C}$, followed by 40 cycles of $15 \mathrm{~s}$ at $94{ }^{\circ} \mathrm{C}$, and $30 \mathrm{~s}$ at $60^{\circ} \mathrm{C}$. The relative expression level of target genes were calculated according to the $2^{-\Delta \Delta C t}$ method as described in Schmittgen and Livak (2008) [54], with JsACTIN2 as an internal referent gene. Three independent experiments were conducted each with pooled flowers from three plants. The gene specific primers (listed in Table S3) were designed by the Primer3+ program (http://www.bioinformatics.nl/cgi-bin/primer3plus/primer3plus.cgi) (access on 5 March 2017). Each primer pair was subjected to melt curve analysis for specificity, and annealing temperature optimization was conducted by testing a range of temperatures from 55 to $70{ }^{\circ} \mathrm{C}$.

For statistical analysis, two-way ANOVA and pair-wide multiple t-tests on means of the three biological replicates were conducted using the GraphPad Prism software (version 7).

\subsection{Transient Expression and Subcellular Localizaton Analysis}

Binary vector pK7FWG2 [55] was used for expression of the coding sequences of JsSWEETs in fusion with a C-terminal eGFP, using the Gateway cloning strategy (see Table S2 for primers used for cloning). A GFP-along expressing plasmid was also constructed for control. Expression from these plasmids was driven by the cauliflower mosaic virus $35 \mathrm{~S}$ promoter. All the constructs were verified by sequencing.

Isolation and transformation of protoplasts from Jasminum petals and Arabidopsis leaves were carried out according to the published protocols [56,57]. For transient expression in Nicotiana benthamiana, Agrobacterium strain GV3101 harboring each expression plasmid was used to infiltrate leaves from four-week-old plants according to method in reference [58].

Fluorescence imaging was realized in a Leica SP8 Confocal Microscope, with a setting for GFP $(E x=488 \mathrm{~nm}, \mathrm{Em}=507$ to $530 \mathrm{~nm})$ and for chlorophyll auto-fluorescence $(E x=488 \mathrm{~nm}, \mathrm{Em}>670 \mathrm{~nm})$. Protoplasts fluorescence were monitored at $16 \mathrm{~h}$ post transformation and Nicotiana leaves were observed at 2 days post infiltration.

\subsection{Yeast Sugar Uptake Complementation Assay}

The coding sequences of JsSWEETs were each subcloned into the yeast expression vector pRS426met25 with the strong promoter MET25 [59] (the cloning primers are listed in Table S2). To measure sugar uptake, we used the yeast mutant strain EBY.VW4000 (MAT $\alpha$ leu2-3,112 ura3-52

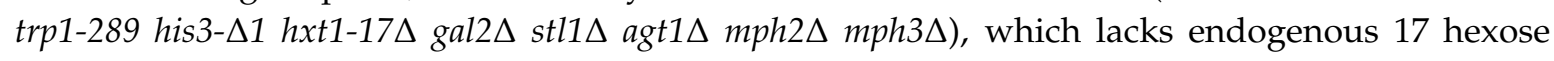
transporters, 1 galactose transporter and 2 maltose permease [44]. Plasmids with or without the JsSWEET CDS were introduced into the yeast using standard PEG protocol, and transformants were selected in synthetic complete (SD) medium without uracil supplemented with $2 \%$ maltose. 
For uptake complementation, the maltose in the SD selection medium was replaced with respective sugar form. The yeast cultures were diluted to an OD600 of 1, and series of 10-fold dilutions were spotted on agar plates. Growth of the yeast cells was monitored after $4-6$ days of incubation at $28^{\circ} \mathrm{C}$.

\subsection{Genebank Accession}

The sequence read archive (SRA) for the RNA-seq described here may be accessed with the numbers SRR9613490 through SRR9613499. Transcriptome of the J. sambac flowers and leaves at anthesis been deposited at DDBJ/ENA/GenBank under the accession GHOY00000000. The JsSWEET sequences in the Ganebank are JsSWEET1 (MN227187), JsSWEET2 (MN227188), JsSWEET5 (MN227189), JsSWEET9 (MN227190), JsSWEET10 (MN227191), JsSWEET16 (MN227193), and JsSWEET17 (MN227192).

Supplementary Materials: The following are available online at http://www.mdpi.com/1422-0067/20/16/4001/s1, Supplemental Figure S1: Protein sequence alignment of the seven JsSWEETs and the Arabidopsis and rice homologs, Supplemental Table S1: List of Primers used for RACE cloning, Supplemental Table S2: List of Primers used for full length PCR amplification, Supplemental Table S3: List of Primers used for RT-qPCR. Supplemental Table S4: Percent Identities of JsSWEETs with homolgous from Arabidopsis and rice.

Author Contributions: Conceptualization, B.W.; Data curation, P.W. (Panpan Wang), P.W. (Peining Wei), F.N., X.L., M.L., Y.Y., and B.W.; Formal analysis, P.W. (Panpan Wang), P.W. (Peining Wei), F.N., X.L., M.L., Y.Y., and B.W.; Funding acquisition, M.L., Y.Y. and B.W.; Investigation, P.W. (Panpan Wang), P.W. (Peining Wei), F.N., X.L., and H.Z.; Methodology, P.W. (Panpan Wang), M.L. and Y.Y.; Project administration, M.L.and B.W.; Resources, H.Z; Supervision, B.W.; Validation, Y.Y.; Visualization, P.W. (Panpan Wang); Writing-original draft, P.W. (Panpan Wang) and B.W.; Writing-review and editing, B.W.

Funding: This research was funded by the Fujian Natural Science Foundation (grant number 2017J0106) and The APC was funded by the College of Horticulture at Fujian Agric. \& Forestry University.

Acknowledgments: We acknowledge E. Boles for providing the yeast strain EBY4000. Wei Wang and Yongyan Zhang are thankful for their technical assistance.

Conflicts of Interest: The authors declare no conflict of interest. The funders had no role in the design of the study; in the collection, analyses, or interpretation of data; in the writing of the manuscript, or in the decision to publish the results.

\section{References}

1. Chen, L.Q.; Cheung, L.S.; Feng, L.; Tanner, W.; Frommer, W.B. Transport of sugars. Annu. Rev. Biochem. 2015, 84, 865-894. [CrossRef] [PubMed]

2. Lalonde, S.; Frommer, W.B. Sut sucrose and mst monosaccharide transporter inventory of the selaginella genome. Front. Plant. Sci. 2012, 3. [CrossRef] [PubMed]

3. Johnson, D.A.; Thomas, M.A. The monosaccharide transporter gene family in arabidopsis and rice: A history of duplications, adaptive evolution, and functional divergence. Mol. Biol. Evol. 2007, 24, 2412-2423. [CrossRef] [PubMed]

4. Buttner, M. The monosaccharide transporter(-like) gene family in arabidopsis. FEBS Lett. 2007, 581, $2318-2324$. [CrossRef] [PubMed]

5. Rottmann, T.; Fritz, C.; Sauer, N.; Stadler, R. Glucose uptake via stp transporters inhibits in vitro pollen tube growth in a hexokinase1-dependent manner in arabidopsis thaliana. Plant. Cell 2018, 30, 2057-2081. [CrossRef]

6. Rottmann, T.; Zierer, W.; Subert, C.; Sauer, N.; Stadler, R. Stp10 encodes a high-affinity monosaccharide transporter and is induced under low-glucose conditions in pollen tubes of arabidopsis. J. Exp. Bot. 2016, 67, 2387-2399. [CrossRef] [PubMed]

7. Yamada, K.; Saijo, Y.; Nakagami, H.; Takano, Y. Regulation of sugar transporter activity for antibacterial defense in arabidopsis. Science 2016, 354, 1427-1430. [CrossRef] [PubMed]

8. Lemonnier, P.; Gaillard, C.; Veillet, F.; Verbeke, J.; Lemoine, R.; Coutos-Thevenot, P.; La Camera, S. Expression of arabidopsis sugar transport protein stp13 differentially affects glucose transport activity and basal resistance to botrytis cinerea. Plant. Mol. Biol. 2014, 85, 473-484. [CrossRef] [PubMed]

9. Riesmeier, J.W.; Willmitzer, L.; Frommer, W.B. Evidence for an essential role of the sucrose transporter in phloem loading and assimilate partitioning. EMBO J. 1994, 13, 1-7. [CrossRef] 
10. Ayre, B.G. Membrane-transport systems for sucrose in relation to whole-plant carbon partitioning. Mol. Plant. 2011, 4, 377-394. [CrossRef]

11. Kuhn, C.; Grof, C.P. Sucrose transporters of higher plants. Curr. Opin. Plant. Biol. 2010, 13, $288-298$. [CrossRef] [PubMed]

12. Durand, M.; Mainson, D.; Porcheron, B.; Maurousset, L.; Lemoine, R.; Pourtau, N. Carbon source-sink relationship in arabidopsis thaliana: The role of sucrose transporters. Planta 2018, 247, 587-611. [CrossRef] [PubMed]

13. Latorraca, N.R.; Fastman, N.M.; Venkatakrishnan, A.J.; Frommer, W.B.; Dror, R.O.; Feng, L. Mechanism of substrate translocation in an alternating access transporter. Cell 2017, 169, 96-107.e12. [CrossRef] [PubMed]

14. Eom, J.S.; Chen, L.Q.; Sosso, D.; Julius, B.T.; Lin, I.W.; Qu, X.Q.; Braun, D.M.; Frommer, W.B. Sweets, transporters for intracellular and intercellular sugar translocation. Curr. Opin. Plant. Biol. 2015, 25, 53-62. [CrossRef] [PubMed]

15. Chen, L.Q.; Qu, X.Q.; Hou, B.H.; Sosso, D.; Osorio, S.; Fernie, A.R.; Frommer, W.B. Sucrose efflux mediated by sweet proteins as a key step for phloem transport. Science 2012, 335, 207-211. [CrossRef] [PubMed]

16. Chen, L.Q.; Hou, B.H.; Lalonde, S.; Takanaga, H.; Hartung, M.L.; Qu, X.Q.; Guo, W.J.; Kim, J.G.; Underwood, W.; Chaudhuri, B.; et al. Sugar transporters for intercellular exchange and nutrition of pathogens. Nature 2010, 468, 527-532. [CrossRef] [PubMed]

17. Tao, Y.; Cheung, L.S.; Li, S.; Eom, J.S.; Chen, L.Q.; Xu, Y.; Perry, K.; Frommer, W.B.; Feng, L. Structure of a eukaryotic sweet transporter in a homotrimeric complex. Nature 2015, 527, 259-263. [CrossRef] [PubMed]

18. Xuan, Y.H.; Hu, Y.B.; Chen, L.Q.; Sosso, D.; Ducat, D.C.; Hou, B.H.; Frommer, W.B. Functional role of oligomerization for bacterial and plant sweet sugar transporter family. Proc. Natl. Acad. Sci. USA 2013, 110, E3685-E3694. [CrossRef] [PubMed]

19. Han, L.; Zhu, Y.; Liu, M.; Zhou, Y.; Lu, G.; Lan, L.; Wang, X.; Zhao, Y.; Zhang, X.C. Molecular mechanism of substrate recognition and transport by the atsweet13 sugar transporter. Proc. Natl. Acad. Sci. USA 2017, 114, 10089-10094. [CrossRef] [PubMed]

20. Xu, Y.; Tao, Y.; Cheung, L.S.; Fan, C.; Chen, L.Q.; Xu, S.; Perry, K.; Frommer, W.B.; Feng, L. Structures of bacterial homologues of sweet transporters in two distinct conformations. Nature 2014, 515, 448-452. [CrossRef] [PubMed]

21. Patil, G.; Valliyodan, B.; Deshmukh, R.; Prince, S.; Nicander, B.; Zhao, M.; Sonah, H.; Song, L.; Lin, L.; Chaudhary, J.; et al. Soybean (glycine max) sweet gene family: Insights through comparative genomics, transcriptome profiling and whole genome re-sequence analysis. BMC Genome 2015, 16, 520. [CrossRef] [PubMed]

22. Gautam, T.; Saripalli, G.; Gahlaut, V.; Kumar, A.; Sharma, P.K.; Balyan, H.S.; Gupta, P.K. Further studies on sugar transporter (sweet) genes in wheat (Triticum aestivum L.). Mol. Biol. Rep. 2019, 46, 2327-2353. [CrossRef] [PubMed]

23. Zhang, W.; Wang, S.; Yu, F.; Tang, J.; Shan, X.; Bao, K.; Yu, L.; Wang, H.; Fei, Z.; Li, J. Genome-wide characterization and expression profiling of sweet genes in cabbage (Brassica oleracea var. Capitata L.) reveal their roles in chilling and clubroot disease responses. BMC Genom. 2019, 20, 93. [CrossRef] [PubMed]

24. Li, W.; Ren, Z.; Wang, Z.; Sun, K.; Pei, X.; Liu, Y.; He, K.; Zhang, F.; Song, C.; Zhou, X.; et al. Evolution and stress responses of gossypium hirsutum sweet genes. Int. J. Mol. Sci. 2018, 19, 769. [CrossRef] [PubMed]

25. Sui, J.L.; Xiao, X.H.; Qi, J.Y.; Fang, Y.J.; Tang, C.R. The sweet gene family in hevea brasiliensis-Its evolution and expression compared with four other plant species. FEBS Open Biol. 2017, 7, 1943-1959. [CrossRef] [PubMed]

26. Jia, B.; Zhu, X.F.; Pu, Z.J.; Duan, Y.X.; Hao, L.J.; Zhang, J.; Chen, L.Q.; Jeon, C.O.; Xuan, Y.H. Integrative view of the diversity and evolution of sweet and semisweet sugar transporters. Front. Plant. Sci. 2017, 8. [CrossRef]

27. Jeena, G.S.; Kumar, S.; Shukla, R.K. Structure, evolution and diverse physiological roles of sweet sugar transporters in plants. Plant. Mol. Biol. 2019, 100, 351-365. [CrossRef]

28. Bezrutczyk, M.; Yang, J.; Eom, J.S.; Prior, M.; Sosso, D.; Hartwig, T.; Szurek, B.; Oliva, R.; Vera-Cruz, C.; White, F.F.; et al. Sugar flux and signaling in plant-microbe interactions. Plant. J.: Cell Mol. Biol. 2018, 93, 675-685. [CrossRef] 
29. Bezrutczyk, M.; Hartwig, T.; Horshman, M.; Char, S.N.; Yang, J.; Yang, B.; Frommer, W.B.; Sosso, D. Impaired phloem loading in zmsweet13a,b,c sucrose transporter triple knock-out mutants in zea mays. New Phytol. 2018, 218, 594-603. [CrossRef]

30. Li, Y.; Wang, Y.; Zhang, H.; Zhang, Q.; Zhai, H.; Liu, Q.; He, S. The plasma membrane-localized sucrose transporter ibsweet10 contributes to the resistance of sweet potato to fusarium oxysporum. Front. Plant. Sci. 2017, 8. [CrossRef]

31. Chen, H.-Y.; Huh, J.-H.; Yu, Y.-C.; Ho, L.-H.; Chen, L.-Q.; Tholl, D.; Frommer, W.B.; Guo, W.-J. The arabidopsis vacuolar sugar transporter sweet2 limits carbon sequestration from roots and restricts pythium infection. Plant. J.: Cell Mol. Biol. 2015, 83, 1046-1058. [CrossRef]

32. Lin, I.W.; Sosso, D.; Chen, L.Q.; Gase, K.; Kim, S.G.; Kessler, D.; Klinkenberg, P.M.; Gorder, M.K.; Hou, B.H.; $\mathrm{Qu}$, X.Q.; et al. Nectar secretion requires sucrose phosphate synthases and the sugar transporter sweet9. Nature 2014, 508, 546-549. [CrossRef] [PubMed]

33. Sosso, D.; Luo, D.; Li, Q.B.; Sasse, J.; Yang, J.; Gendrot, G.; Suzuki, M.; Koch, K.E.; McCarty, D.R.; Chourey, P.S.; et al. Seed filling in domesticated maize and rice depends on sweet-mediated hexose transport. Nat. Genet. 2015, 47, 1489-1493. [CrossRef] [PubMed]

34. Yang, J.L.; Luo, D.P.; Yang, B.; Frommer, W.B.; Eom, J.S. Sweet11 and 15 as key players in seed filling in rice. New Phytol. 2018, 218, 604-615. [CrossRef]

35. Zhang, Z.; Zou, L.; Ren, C.; Ren, F.; Wang, Y.; Fan, P.; Li, S.; Liang, Z. Vvsweet10 mediates sugar accumulation in grapes. Genes 2019, 10, 255. [CrossRef] [PubMed]

36. Borghi, M.; Fernie, A.R. Floral metabolism of sugars and amino acids: Implications for pollinators' preferences and seed and fruit set. Plant. Physiol. 2017, 175, 1510-1524. [CrossRef]

37. Guan, Y.F.; Huang, X.Y.; Zhu, J.; Gao, J.F.; Zhang, H.X.; Yang, Z.N. Ruptured pollen grain1, a member of the $\mathrm{mtn} 3 /$ saliva gene family, is crucial for exine pattern formation and cell integrity of microspores in arabidopsis. Plant. Physiol. 2008, 147, 852-863. [CrossRef]

38. Ge, Y.X.; Angenent, G.C.; Dahlhaus, E.; Franken, J.; Peters, J.; Wullems, G.J.; Creemers-Molenaar, J. Partial silencing of the nec1 gene results in early opening of anthers in petunia hybrida. Mol. Genet. Genom. Mgg 2001, 265, 414-423.

39. Deng, Y.M.; Sun, X.B.; Gu, C.S.; Jia, X.P.; Liang, L.J.; Su, J.L. Identification of pre-fertilization reproductive barriers and the underlying cytological mechanism in crosses among three petal-types of Jasminum sambac and their relevance to phylogenetic relationships. PLoS ONE 2017, 12. [CrossRef]

40. Deng, Y.M.; Jia, X.P.; Liang, L.J.; Gu, C.S.; Sun, X.B. Morphological anatomy, sporogenesis and gametogenesis in flowering process of jasmine (Jasminum sambac aiton). Sci. Hortic.-Amst. 2016, 198, 257-266. [CrossRef]

41. Bera, P.; Mukherjee, C.; Mitra, A. Enzymatic production and emission of floral scent volatiles in Jasminum sambac. Plant. Sci 2017, 256, 25-38. [CrossRef] [PubMed]

42. Ge, Y.X.; Angenent, G.C.; Wittich, P.E.; Peters, J.; Franken, J.; Busscher, M.; Zhang, L.M.; Dahlhaus, E.; Kater, M.M.; Wullems, G.J.; et al. Nec1, a novel gene, highly expressed in nectary tissue of petunia hybrida. Plant. J.: Cell Mol. Biol. 2000, 24, 725-734. [CrossRef]

43. Wieczorke, R.; Krampe, S.; Weierstall, T.; Freidel, K.; Hollenberg, C.P.; Boles, E. Concurrent knock-out of at least 20 transporter genes is required to block uptake of hexoses in saccharomyces cerevisiae. FEBS Lett. 1999, 464, 123-128. [CrossRef]

44. Sun, L.; Sui, X.; Lucas, W.J.; Li, Y.; Feng, S.; Ma, S.; Fan, J.; Gao, L.; Zhang, Z. Down-regulation of the sucrose transporter cssut1 causes male sterility by altering carbohydrate supply. Plant. Physiol. 2019, 180, 986-997. [CrossRef] [PubMed]

45. Wang, S.; Yokosho, K.; Guo, R.; Whelan, J.; Ruan, Y.L.; Ma, J.F.; Shou, H. The soybean sugar transporter gmsweet15 mediates sucrose export from endosperm to early embryo. Plant. Physiol. 2019, 180, 2133-2141. [CrossRef] [PubMed]

46. Chardon, F.; Bedu, M.; Calenge, F.; Klemens, P.A.W.; Spinner, L.; Clement, G.; Chietera, G.; Leran, S.; Ferrand, M.; Lacombe, B.; et al. Leaf fructose content is controlled by the vacuolar transporter sweet17 in arabidopsis. Curr. Biol. 2013, 23, 697-702. [CrossRef] [PubMed]

47. Guo, W.J.; Nagy, R.; Chen, H.Y.; Pfrunder, S.; Yu, Y.C.; Santelia, D.; Frommer, W.B.; Martinoia, E. Sweet17, a facilitative transporter, mediates fructose transport across the tonoplast of arabidopsis roots and leaves. Plant. Physiol. 2014, 164, 777-789. [CrossRef] 
48. Klemens, P.A.; Patzke, K.; Deitmer, J.; Spinner, L.; Le Hir, R.; Bellini, C.; Bedu, M.; Chardon, F.; Krapp, A.; Neuhaus, H.E. Overexpression of the vacuolar sugar carrier atsweet16 modifies germination, growth, and stress tolerance in arabidopsis. Plant. Physiol. 2013, 163, 1338-1352. [CrossRef]

49. Bock, K.W.; Honys, D.; Ward, J.M.; Padmanaban, S.; Nawrocki, E.P.; Hirschi, K.D.; Twell, D.; Sze, H. Integrating membrane transport with male gametophyte development and function through transcriptomics. Plant. Physiol. 2006, 140, 1151-1168. [CrossRef]

50. Engel, M.L.; Davis, R.H.; McCormick, S. Green sperm. Identification of male gamete promoters in arabidopsis. Plant. Physiol. 2005, 138, 2124-2133. [CrossRef]

51. Grabherr, M.G.; Haas, B.J.; Yassour, M.; Levin, J.Z.; Thompson, D.A.; Amit, I.; Adiconis, X.; Fan, L.; Raychowdhury, R.; Zeng, Q.; et al. Full-length transcriptome assembly from rna-seq data without a reference genome. Nat. Biotechnol. 2011, 29, 644-652. [CrossRef] [PubMed]

52. Wang, L.; Feng, Z.; Wang, X.; Wang, X.; Zhang, X. Degseq: An r package for identifying differentially expressed genes from rna-seq data. Bioinformatics 2010, 26, 136-138. [CrossRef] [PubMed]

53. Young, M.D.; Wakefield, M.J.; Smyth, G.K.; Oshlack, A. Gene ontology analysis for rna-seq: Accounting for selection bias. Genome Biol. 2010, 11, R14. [CrossRef] [PubMed]

54. Schmittgen, T.D.; Livak, K.J. Analyzing real-time pcr data by the comparative c(t) method. Nat. Protoc. 2008, 3, 1101-1108. [CrossRef] [PubMed]

55. Karimi, M.; Inze, D.; Depicker, A. Gateway vectors for agrobacterium-mediated plant transformation. Trends Plant. Sci. 2002, 7, 193-195. [CrossRef]

56. Shen, J.; Fu, J.; Ma, J.; Wang, X.; Gao, C.; Zhuang, C.; Wan, J.; Jiang, L. Isolation, culture, and transient transformation of plant protoplasts. Curr. Protoc. Cell Biol. 2014, 63, 2.8.1-2.8.17.

57. Yoo, S.D.; Cho, Y.H.; Sheen, J. Arabidopsis mesophyll protoplasts: A versatile cell system for transient gene expression analysis. Nat. Protoc. 2007, 2, 1565-1572. [CrossRef]

58. Li, X.; Chanroj, S.; Wu, Z.; Romanowsky, S.M.; Harper, J.F.; Sze, H. A distinct endosomal ca2+/mn2+ pump affects root growth through the secretory process. Plant. Physiol. 2008, 147, 1675-1689. [CrossRef]

59. Mumberg, D.; Muller, R.; Funk, M. Regulatable promoters of saccharomyces cerevisiae: Comparison of transcriptional activity and their use for heterologous expression. Nucleic Acids Res. 1994, 22, 5767-5768. [CrossRef]

(C) 2019 by the authors. Licensee MDPI, Basel, Switzerland. This article is an open access article distributed under the terms and conditions of the Creative Commons Attribution (CC BY) license (http://creativecommons.org/licenses/by/4.0/). 